\title{
Energy Filtered Scanning Confocal Electron Microscopy in a Double Aberration-Corrected Transmission Electron Microscope
}

\author{
P.Wang*, G. Behan*, A.I. Kirkland*, and P.D. Nellist* \\ Department of Materials, University of Oxford, Parks Road, Oxford OX1 3PH, UK
}

With the development of spherical aberration correctors for both transmission electron microscopy (TEM) and scanning transmission electron microscopy (STEM), the spatial resolution in 2D images has been dramatically improved [1]. This is because the correction of spherical aberration allows a larger probe forming aperture thus forming a finer probe. In addition to increasing the lateral resolution, a larger convergence angle also leads to a reduced depth of field, which is likely to approach a few nanometres with the latest aberration-corrected microscopes. Having such a narrow depth of field offers the feasibility of depth sectioning of samples in similar fashion to confocal scanning optical microscopy (CSOM). It has been shown that spherical aberration corrected TEM/STEM instruments are capable of working in this confocal mode [2], which has previously be named as scanning confocal electron microscopy (SCEM) [3]

Cosgriff [4] and D'Alfonso [5] have theoretically examined the contrast mechanisms for SCEM using elastically and inelastically scattered electrons, respectively on the identification and location of isolated impurities. Their work showed elastic SCEM suffers no first-order contrast transfer so that image contrast is weak. However, inelastic SCEM or energy-filtered SCEM (EFSCEM) gives improved depth selectivity which allows 3D structure determination. In particular they showed that the transfer function does not have a missing cone, so that even laterally extended or planar objects can be located in depth.

In a previous publication [2], we demonstrated that a confocal electron optical configuration could be achieved in an instrument fitted with two spherical aberration correctors for elastically scattered electrons. The instrument used for those, experiments, the Oxford-JEOL 2200MCO, is also fitted with an in-column energy filter, allowing the possibility of EFSCEM experiments. A difficulty, however, is the residual uncorrected chromatic aberration, which changes the focal length of the post-specimen optics for the inelastic electrons. We have devised a technique whereby the instrument is aligned for the elastic electrons, and the accelerating voltage is then increased by half the value of the energy-loss of interest. As shown in Fig. 1, this result in confocal configuration for electrons of the desired energy-loss, but with the confocal point shifted down. Figure 2 shows the energy dispersion plane in the confocal configuration, and shows how focus is regained at the desired energy loss. The effect of moving the sample out of the confocal plane is shown in Fig. 3. Because we are only detecting electrons with an energy that is characteristic of inelastic scattering in the sample, when the sample is out of the confocal plane the scattering no longer forms a focused spot. The confocal image is formed by summing the electrons that are accurately focused, and so moving the sample out of the confocal plane reduces the signal. It is exactly that reduction that allows the three-dimensional information to be measured, and we have performed experiments to measure the resulting EFSCEM depth resolution [6].

References

[1] P. D. Nellist et al., Science 305, 1741 (2004).

[2] P. D. Nellist et al., Applied Physics Letters 89, 124105 (2006). 
[3] S. P. Frigo, et al, Applied Physics Letters 81, 2112 (2002).

[4] E. C. Cosgriff et al., Ultramicroscopy 108, 1558 (2008).

[5] A. J. D'Alfonso et al., Ultramicroscopy 108, 1567 (2008).

[6] Manuscript in preparation.

[7] The authors would like to acknowledge the support of the Leverhulme Trust, JEOL UK, the EPSRC and the Department of Materials at the University of Oxford

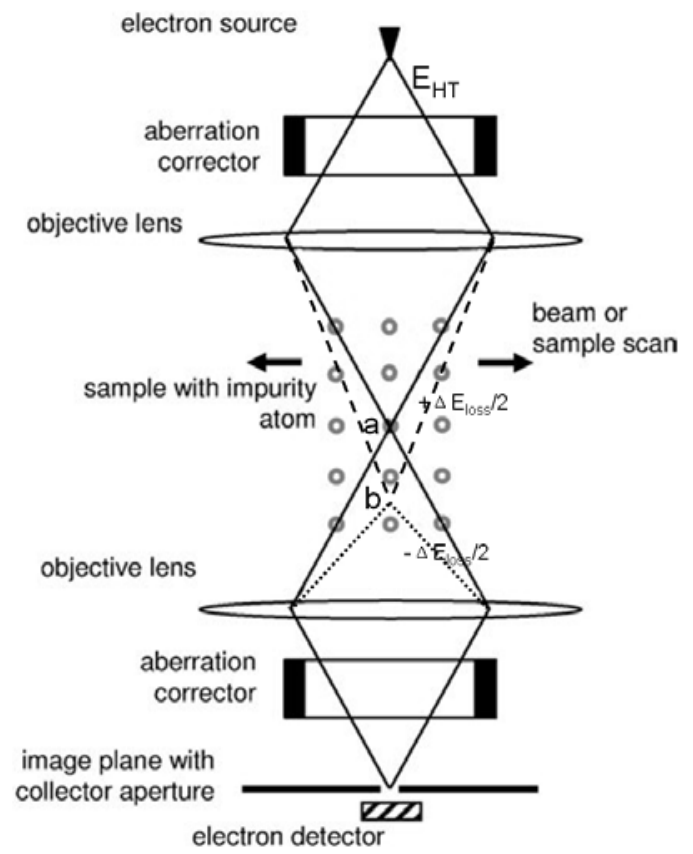

Figure 1 Schematic diagram of the SCEM optics configurations for elastically (solid line) and inelastically scattered electrons of the desired energyloss of $\Delta \mathrm{E}_{\text {loss }}$ (dashed line), respectively. The optics configuration of energyfiltered SCEM for electron energy loss of $\Delta \mathrm{E}_{\text {loss }}$ is established by applying an increment of $\Delta \mathrm{E}_{\mathrm{HT}}=1 / 2 \Delta \mathrm{E}_{\text {loss }}$ to the accelerating voltage $\mathrm{E}_{\mathrm{HT}}$,

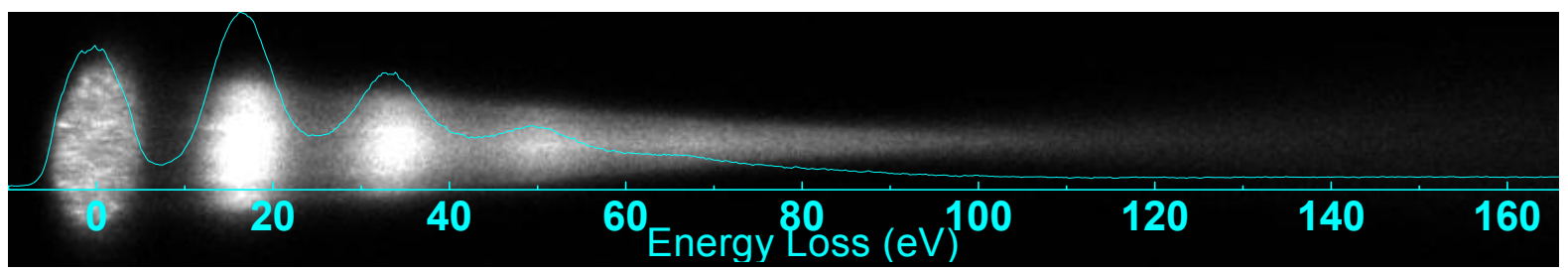

Figure 2 shows the energy dispersion plane in the confocal configuration, and how focus is regained at the desired energy loss of Si L-edge.

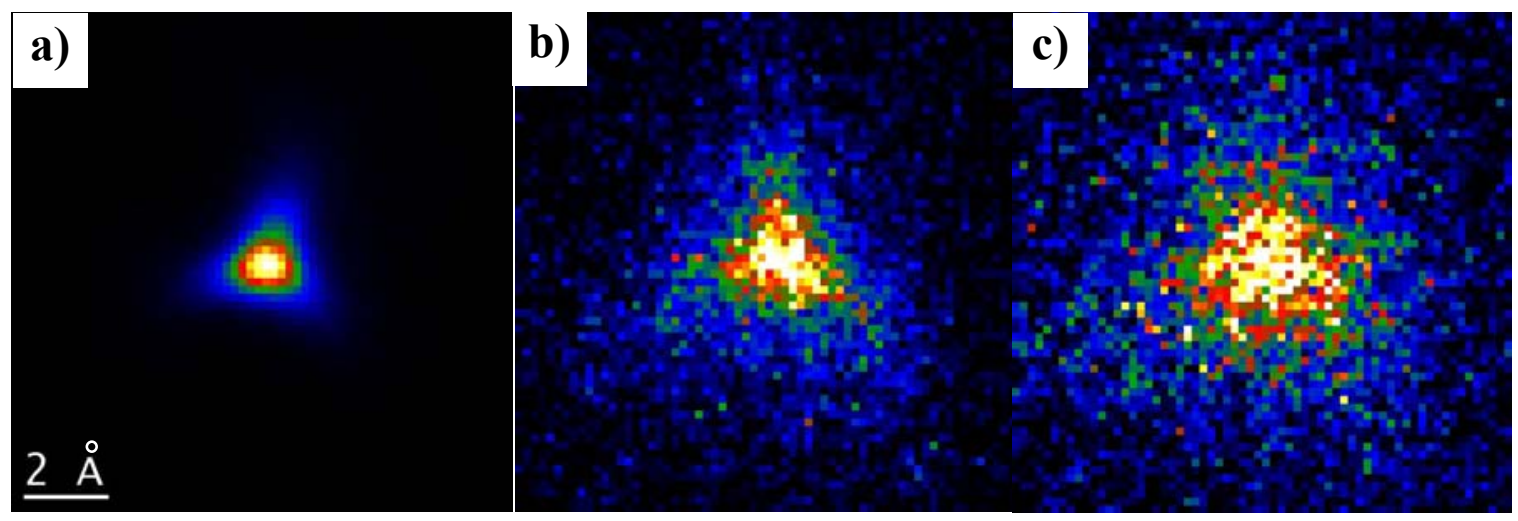

Figure 3 a) shows experimental images of the probe in the elastic confocal mode formed through vacuum (a) and through a carbon film inelastic confocal mode with $5 \mathrm{eV}$ energy filtering window placed at the onset of carbon K-edge, when the film is just at (b) and 56nm over the confocal point (c), respectively 